Cahiers $d u$ MONDE RUSSE

\section{Cahiers du monde russe}

Russie - Empire russe - Union soviétique et États indépendants

$52 / 4 \mid 2011$

Varia

\title{
Malte Griesse, Communiquer, juger et agir sous Staline
}

\section{Magali Delaloye}

\section{OpenEdition}

\section{Journals}

Édition électronique

URL : http://journals.openedition.org/monderusse/7629

DOI : 10.4000/monderusse.7629

ISSN : $1777-5388$

\section{Éditeur}

Éditions de l'EHESS

\section{Édition imprimée}

Date de publication : 20 décembre 2011

Pagination : 759-762

ISBN : 978-2-7132-2353-2

ISSN : $1252-6576$

Référence électronique

Magali Delaloye, "Malte Griesse, Communiquer, juger et agir sous Staline », Cahiers du monde russe [En ligne], 52/4 | 2011, mis en ligne le 03 décembre 2012, Consulté le 23 septembre 2020. URL : http:// journals.openedition.org/monderusse/7629; DOI : https://doi.org/10.4000/monderusse.7629

Ce document a été généré automatiquement le 23 septembre 2020

(c) École des hautes études en sciences sociales 


\title{
Malte Griesse, Communiquer, juger et agir sous Staline
}

\author{
Magali Delaloye
}

\section{RÉFÉRENCE}

Malte GRIESSE, Communiquer, juger et agir sous Staline. La personne prise entre ses liens avec les proches et son rapport au système politico-idéologique.

Francfort-sur-le-Main : Peter Lang, 2011, 536 p.

1 L'imposant ouvrage de Malte Griesse est une contribution des plus importantes au mouvement historiographique qui s'est développé ces dernières années, à la suite de l'ouverture des archives soviétiques de la période stalinienne, et qui propose une approche renouvelée des recherches menées en leur temps par les tenants de l'« école révisionniste». De nombreuses études sur la subjectivité des individus en Union soviétique ont vu le jour grâce également aux apports des théories foucaldiennes ${ }^{1}$. Elles ont permis de mieux cerner les mécanismes et les niveaux d'adhésion des citoyens à un régime brutal. Or, le présent ouvrage, que l'auteur a tiré de sa thèse de doctorat, n'est pas seulement une contribution parmi d'autres à cette réflexion historiographique. Il s'inscrit dans ce mouvement par son étude des relations verticales qui lient l'acteur et le régime, mais il ajoute à son analyse une dimension horizontale, celle des relations qui unissent un acteur à son entourage. Le cœur de l'analyse porte alors sur les pratiques de communication des acteurs, tant avec eux-mêmes qu'avec leurs proches: c'est par elles que s'accomplit le «travail sur soi » et se construit la subjectivité de la personne.

2 L'auteur a choisi d'étudier d'anciens bolcheviks à travers leurs ego-documents (auto)biographies, journaux personnels et correspondances. Engagés dans le mouvement social-démocrate dès les premières heures, ayant souvent constitué leurs réseaux personnels dans un engagement militant sous la bannière du parti, non seulement ils adhèrent complètement aux valeurs de la révolution, mais aussi, ayant 
risqué leur vie pour la cause, ils s'estiment fondateurs du nouvel État et s'identifient avec lui.

3 Pour saisir la complexité et la richesse des documents personnels, l'auteur combine trois approches différentes (chapitre 2). Dans son analyse des ego-documents, il reprend d'abord l'apport de Foucault sur les techniques de soi, les rapports du sujet à lui-même et au système de pouvoir et sur le potentiel de subversion de l'acteur dans ses relations avec le régime. Une deuxième source d'inspiration méthodologique de l'auteur est Hanna Arendt, moins pour son concept de totalitarisme que pour sa conception de l'agir. Chez Arendt, l'agir humain ne peut se réaliser que dans le cadre des interactions où les acteurs produisent leur identité. Seuls, ils font face à une multitude d'identités et c'est dans le contact avec les autres qu'ils trouvent une cohérence à leur soi. Enfin, le troisième outil est inspiré par la sociologie de la critique, qui propose notamment une solution pour combiner les dimensions macrosociale (la personne face aux rapports de force qu'impose le régime) et microsociale (la personne agissant dans son entourage proche).

Griesse présente d'abord la pratique du mimétisme (podraženie) comme mode d'apprentissage du travail sur soi pour créer l'Homme nouveau (chapitre 4). Outre les débats sur l'éducation et l'auto-éducation, l'analyse porte sur le rapport que les acteurs ont vis-à-vis de la variété de modèles proposés par le régime. Ces modèles peuvent être tant des figures réelles - par exemple le père ou Lenin - que des figures fictionnelles les héros du réalisme socialiste. Dès le milieu des années trente, de vieux bolcheviks de haut vol qui servent d'exemple pour le mimétisme disparaissent dans les purges, déchus de la qualité de bolchevik modèle. Cela provoque chez les acteurs un sentiment d'abandon, renforcé par l'impossibilité de parler à quiconque de son désarroi, mais aussi le soupçon envers le régime.

Deuxième pratique étudiée : l'imposture (chapitre 6). Cette pratique n'est pas nouvelle dans l'histoire de la Russie et la révolution a fait naitre sa cohorte d'imposteurs. Ces derniers ne le sont pas seulement par intérêt, mais aussi pour masquer des tares dans leur biographie qui les empêchent de s'insérer dans le nouveau tissu social. Le point commun de tous les imposteurs est qu'ils doivent trouver le moyen de stabiliser leur identité dans l'imposture pour ne pas avoir à en changer constamment. Cela signifie qu'ils doivent intérioriser leur fausse identité, cette dernière finissant par prendre le dessus, jusque dans la communication intime. L'auteur nous présente une large palette de cas d'imposture: celui de Leonid Krasin, commissaire du peuple au Commerce extérieur, au double langage ; celui, bien connu, de Malinovskij et de Frère Gapon, qui a suscité la hantise de la trahison chez les communistes engagés ; celui de K.A. Koškin, ex-menchevik dissimulé; enfin, celui de Stepan Podlubnyj, ex-koulak déporté à la brillante carrière au sein du NKVD.

6 Un cas illustre les glissements possibles entre mimétisme et imposture : celui du graphomane A.G.Solov'ev (chapitre 5). Dans les écrits autobiographiques qu'il retravaille sa vie durant, il présente le travail qu'il accomplit sur lui-même en vue de s'améliorer. Ce récit passe par la description de nombreuses rencontres avec des mentors. Or, la frontière entre la réalité et la fiction est souvent franchie dans ses notes sur ces personnes. Ainsi, dans sa construction mimétique, il glisse vers l'imposture.

7 Pour les acteurs historiques, communiquer clarifie ce qui, s'il n'était pas énoncé, resterait dans l'ombre : la communication est le mécanisme qui permet de faire entrer leurs pensées dans la lumière et, ainsi de créer des opinions, en particulier sur le 
régime dans lequel les acteurs vivent. L'auteur montre que c'est justement par la communication avec leur entourage que les anciens bolcheviks peuvent se positionner et que peut naître et se développer chez eux la critique du régime stalinien.

Or, le régime reste très ambigu face à la parole de ses citoyens : d'un côté, il impose à la population le tabou sur les événements en cours; de l'autre, il les pousse à parler beaucoup. Griesse pose l'hypothèse, brillamment démontrée, que «le régime stalinien avait profondément besoin de l'opinion (et du soutien actif) de la population [...], mais qu'il en avait encore plus profondément peur à cause du potentiel critique qu'incarnait l'opinion, au-delà de (et en parallèle avec) sa capacité mobilisatrice » (p. 241). Ce paradoxe permet d'expliquer que le régime cherche à contrôler à tout prix les espaces où la parole est délivrée.

Plusieurs exemples montrent les processus de construction de la critique ou de l'adhésion au régime chez les anciens bolcheviks dans la communication intime: la relation entre Valerian Kujbyšev et sa seconde épouse, Ol'ga Laževa (chapitre 7); la relation, asymétrique, qui lie Nadežda Krupskaja et Alisa Radčenko, sa proche collaboratrice au commissariat du peuple à l'Éducation (chapitre 8) ; A.G. Man'kov, issu d'une famille aisée de l'intelligentsia prérévolutionnaire (chapitre 9). Enfin, la famille d'anciens bolcheviks Anna Kravčenko et Aleksandr Spunde est un cas exceptionnel non seulement en raison de la grande quantité de documents à disposition, mais surtout du fait qu'il montre en détail leur engagement et le besoin quasi viscéral de le partager avec les autres membres de la famille, malgré les risques de censure (perljustracija). Ce fort engagement a pour conséquence de créer des espaces qui peuvent faire naître la critique et provoquer des prises de distance avec des valeurs, par exemple dans l'éducation des enfants (chapitre 10).

10 L'ouvrage se termine par une synthèse qui, à partir de l'analyse sur la formation de l'opinion et le positionnement des acteurs dans leur entourage, propose une vision renouvelée du fonctionnement du système stalinien. Du côté du régime, les espaces communicationnels réduits que les acteurs parviennent à construire et d'où émerge potentiellement la critique représentent un danger qu'il craint de devoir affronter. Son discours reconstruit, notamment de la révolution d'Octobre, ne coïncide plus avec les expériences des acteurs qui y étaient engagés, provoquant chez les vétérans le sentiment que l'État propose une image mensongère. De là naît le doute, voire la critique. Les vétérans partent en " exil intérieur ", en construisant un mur entre leurs pratiques politiques et quotidiennes et leurs pensées profondes. Pour le régime stalinien, de telles pratiques se révèlent nettement moins dangereuses que les nombreux micro-espaces où les acteurs «entrouvrent la porte » de leurs pensées intimes.

11 Cet ouvrage est un apport important à l'historiographie actuelle sur la période stalinienne. Cette recherche, très érudite, proposant des analyses fines et détaillées, vient nourrir les débats sur la nature du régime stalinien et sur son rapport aux sujets. Malgré la précision du livre, on rencontre quelques raccourcis. Par exemple, quand l'auteur écrit que l'idéal androgyne de la révolutionnaire est bien ancré dans les représentations des bolcheviks : l'ampleur de cet ancrage a été largement remis en question par les historiennes du genre ${ }^{2}$. De plus, cet ouvrage a les défauts de ses qualités. La densité des informations et des réflexions masque parfois le fil rouge de la démonstration et l'originalité du livre. On peut également regretter, comme c'est souvent le cas, un travail d'édition assez peu précis, qui a laissé passer un certain 
nombre de coquilles. Mais ces points ne gâchent en rien l'extrême richesse de la réflexion. C'est surtout l'apport empirique qui se révèle le plus passionnant. En nous faisant entrer dans la vie intime de ces anciens bolcheviks, en nous dévoilant leurs doutes, leur capacité de critique et le cheminement de leur positionnement par rapport à leurs proches comme au régime, Griesse a brillamment démontré que l'analyse des pratiques communicatives est fondamentale pour comprendre le régime stalinien.

\section{NOTES}

1. Igal Halfin, Terror in my Soul: Communist Autobiographies on Trial, Cambridge, MA : Harvard University Press, 2003 ; Jochen Hellbeck, Revolution on my Mind: Writing a Diary under Stalin, Cambridge, MA : Harvard University Press, 2006; Brigitte Studer, Heiko Haumann, éds., Stalinistische Subjekte: Individuum und System in der Sowjetunion und der Komintern, 1929-1953, Zürich : Chronos, 2006.

2. Cf., par exemple, Carmen Scheide, Kinder, Küche, Kommunismus: Das Wechselverhältnis zwischen Alltagsleben und Politik am Beispiel Moskauer Arbeiterinnen während der NEP, 1921-1930, Zürich, 2002, Basler Studien zur Kulturgeschichte Osteuropas, Bd. 3. 